\title{
Survival benefit of re-irradiation in esophageal Cancer patients with Locoregional recurrence: a propensity score-matched analysis
}

Liang Hong ${ }^{1 \dagger}$, Yun-xia Huang ${ }^{1 \dagger}$, Qing-yang Zhuang ${ }^{1}$, Xue-qing Zhang ${ }^{1}$, Li-rui Tang ${ }^{1}$, Kai-xin Du², Xiao-yi Lin², Bu-hong Zheng ${ }^{1}$, Shao-li Cai ${ }^{3}$, Jun-xin $\mathrm{Wu}^{1}$ and Jin-luan $\mathrm{Li}^{i^{*}}$ (i)

\begin{abstract}
Background: To investigate the treatment failure pattern and factors influencing locoregional recurrence of esophageal squamous cell carcinoma (ESCC) and examine patient survival with re-irradiation (re-RT) after primary radiotherapy.

Methods: We retrospectively analyzed 87 ESCC patients treated initially with radiotherapy. Failure patterns were classified into regional lymph node recurrence only (LN) and primary failure with/without regional lymph node recurrence (PF). Patients received either re-RT or other treatments (non-re-RT group). Baseline covariates were balanced by a propensity score model. Overall survival (OS) and toxicities were assessed as outcomes.

Results: The median follow-up time was 87 months. Thirty-nine patients received re-RT. Failure pattern and re-RT were independent prognostic factors for OS ( $P=0.040$ and 0.015$)$ by Cox multivariate analysis. Re-RT with concomitant chemotherapy showed no survival benefit over re-RT alone $(P=0.70)$. No differences in characteristics were found between the groups by Chi-square tests after propensity score matching. The Cox model showed that failure pattern and re-RT were prognostic factors with hazard ratios (HR) of $0.319(P=0.025)$ and $0.375(P=0.002)$, respectively, in the matched cohort. Significant differences in OS were observed according to failure pattern $(P=0.004)$ and re-RT $(P<0.001)$. In the re-RT and non-re-RT groups, 9.09\% and 3.03\% of patients experienced tracheoesophageal fistulas, and 15.15\% and $3.03 \%$ of patients developed pericardial/pleural effusion, respectively $(P>0.05)$. The incidence of radiation pneumonitis was higher in the re-RT group (24.24\% vs. 6.06\%, $P=0.039)$, but no cases of pneumonia-related death occurred.

Conclusions: Re-RT improved long-term survival in patients with locoregional recurrent ESCC. Despite a high incidence of radiation pneumonitis, toxicities were tolerable.
\end{abstract}

Keywords: Esophageal squamous cell carcinoma, Locoregional recurrence, Re-irradiation, Propensity score-matched analysis, Overall survival

\section{Background}

Locoregional recurrence is the most common mode of failure in esophageal cancer treated initially with chemoradiotherapy (CRT) and/or surgery [1]. The local recurrence rate after definitive CRT has ranged from 40 to $60 \%$ with a low 5 -year survival rate upon recurrence $[2,3]$. To date,

\footnotetext{
* Correspondence: lijinluan@pku.org.cn

Accepted by ASTRO Annual Meeting, 2018, San Antonio, abstract \#23681

+Liang Hong and Yun-xia Huang contributed equally to this work.

'Department of Radiation Oncology, Fujian Medical University Cancer

Hospital, Fujian Cancer Hospital, Fuzhou 350014, China

Full list of author information is available at the end of the article
}

there is no consensus regarding a curative treatment, leaving limited treatment options for patients with locoregional recurrence esophageal squamous cell carcinoma (ESCC) after CRT.

Chemotherapy is preferred as a systemic treatment for multiple-site recurrence or distant metastasis, whereas definitive local therapy is suitable for locoregional recurrent ESCC with the goal of improving prognosis. Although salvage surgery has curative potential, studies have reported high rates of pulmonary complications $(17-30 \%)$, anastomotic leakage (17-39\%), intensive care

(c) The Author(s). 2018 Open Access This article is distributed under the terms of the Creative Commons Attribution 4.0 International License (http://creativecommons.org/licenses/by/4.0/), which permits unrestricted use, distribution, and 
unit admission (17-22\%), and postoperative mortality (3-15\%) with salvage surgery for locoregional recurrent ESCC after definitive CRT $[4,5]$. These limit the number of patients who are candidates for salvage surgery.

Advancements in radiotherapy have allowed conformal radiation dose distribution with delivery of incremental doses to tumors and a minimal dose to adjacent critical structures. Re-irradiation has shown satisfactory clinical outcome in certain recurrent tumors such as lung cancer, head and neck cancer, high-grade glioma, and rectal cancer [6-11]. In the present study, we evaluated the clinical prognostic factors associated with overall survival (OS) in recurrent ESCC. Propensity score-matched (PSM) analysis was applied to assess clinical outcomes and toxicities of re-RT for locoregional recurrent ESCC to correct for the baseline covariates.

\section{Methods}

\section{Patients}

In the current study, we retrospectively examined 87 consecutive ESCC patients with locoregional recurrence who were admitted to Fujian Cancer Hospital between June 2000 and June 2014. All included patients met the following criteria: a) pathological confirmation of primary ESCC at initial diagnosis; b) a history of initial radiation; c) histological and/or PET-CT confirmation of locoregional recurrence including regional lymph node recurrence only (LN) or primary failure with/without regional lymph node recurrence (PF); d) no evidence of esophageal perforation or ulcer; and e) adequate liver, kidney, and bone marrow functioning with a Karnofsky performance status (KPS) score $\geq 70$. The exclusion criteria were as follows: a) history of other malignancies; b) distant metastases; and c) confirmation of recurrence within 3 months of initial treatment.

Clinical staging at first diagnosis was determined by chest computed tomography (CT) and barium esophagram and/ or endoscopic ultrasound (EUS). Re-staging of initial ESCC was done according to the 8th edition of American Joint Committee on Cancer (AJCC). The current study was approved by the Ethics Committee of Fujian Medical University Cancer Hospital, Fuzhou, China (KT2018-006-01). Because this was a retrospective study involving patient medical records, the requirement of patients' consent was waived.

\section{Treatment}

For initial treatment, 11 (12.6\%) patients received radical resection with adjuvant radio (chemo) therapy (median dose $=52$ Gy, range 40-56 Gy). Thirty-nine (44.8\%) and 37 (42.6\%) patients received CRT and RT, respectively. Among the $43(49.4 \%)$ cases initially treated with chemotherapy (median of 3 cycles, range 1-6 cycles), 30
(34.5\%) received cisplatin and paclitaxel, whereas the remaining $13(14.9 \%)$ received cisplatin or oxaliplatin.

Patients were treated with 6- or 10-MV linear accelerators for initial radiotherapy with 1.8-2.2 Gy/fraction and 5 fractions/week. Initial RT was conventional two-dimensional (36.8\%) or conformal three-dimensional (63.2\%) RT with a median dose of 62 Gy (range 40-76 Gy). The median dose of re-RT was 50 Gy (ranged 21-70 Gy) with 2Gy (range 1.8-4 Gy) per fraction. Intensity-modulated RT $(56.4 \% ; 22 / 39)$ and conformal three-dimensional RT (43.6\%; 17/39) were employed for re-RT. Among the 39 patients treated with re-RT, 19 patients $(48.7 \%)$ received concomitant chemotherapy, of which 6 received cisplatin, 4 received 5-flurouracil (5-FU), and 9 received cisplatin combined with 5 -FU. The remaining 20 (51.3\%) patients received RT alone.

The biological effectiveness of radiation schedule was calculated by the biologically effective dose (BED) formula: $B E D=n \times d(1+d /(\alpha / \beta)), d$ for the dose per fraction (Gy) and $\mathrm{n}$ for the number of fractions. Assuming an $\alpha / \beta$ ratio of 10 Gy for ESCC $\left(B D_{10}\right)$ [12]. For re-RT patients, the cumulative dose was calculated.

For patients without re-RT, 7 (8.0\%) patients received chemotherapy alone with cisplatin combined with 5-FU, whereas 3 (3.4\%) patients underwent salvage total esophagectomy with gastric pull-through. The remaining 38 (43.7\%) patients received supportive care including esophageal stenting, dilation or percutaneous endoscopic gastrostomy to relieve dysphagia.

\section{Follow-up}

The primary endpoint was OS, which was defined as the time duration from recurrence diagnosis to death or last follow-up. The recurrence-free interval (RFI) was defined as the time interval from the end of initial treatment to the recurrence diagnosis. According to the National Cancer Institute Common Terminology Criteria for Adverse Events (CTCAE) version 4.0, toxicities recorded in the patients' medical records were retrospectively graded [13]. Tracheoesophageal fistula (TEF), pericardial/pleural effusion, and radiation pneumonitis (RP) were recorded.

\section{Statistical analysis}

All statistical tests were performed using SPSS version 22.0 (IBM Corporation, Armonk, NY, USA). The propensity score matching ratio was set to $1: 1$ to minimize differences due to age, gender, primary tumor location, and initial clinical stage. Chi-square $\left(x^{2}\right)$ and Fisher's exact tests were applied to compare unmatched background factors. Survival curves were constructed and compared by the Kaplan-Meier method and log-rank tests. The Cox regression model was employed for the univariate analysis and multivariate analysis. $P$-values $<0.05$ were considered statistically significant. 
Table 1 Characteristics of 87 patients with locoregional recurrent esophageal cancer

\begin{tabular}{lll}
\hline Variables & Number & Percent \\
\hline $\begin{array}{l}\text { Age (years) } \\
<65\end{array}$ & 58 & \\
$\geq 65$ & 29 & 36.7 \\
KPS & & \\
$70-80$ & 32 & 36.8 \\
$>80$ & 55 & 63.2 \\
Gender & & \\
Male & 65 & 74.7 \\
Female & 22 & 25.3 \\
Smoking & & \\
Yes & 31 & 35.6 \\
No & 56 & 64.4
\end{tabular}

Alcohol consumption

Yes

No

Primary tumor location

Upper thoracic

Middle and lower thoracic

Initial clinical stage

I+ II

32.9

III

Initial treatment

Surgery + adjuvant radio(chemo)therapy
Definitive chemoradiotherapy
Definitive radiotherapy

Pattern of recurrence

Regional lymph node recurrence only

Local failure

Both

Radiation dose in the initial treatment (Gy)

$$
\leq 50
$$$$
>50
$$

Recurrence-free interval (months)

$$
\leq 12
$$$$
>12
$$

Chemotherapy after recurrence

Yes

No

Re-RT after recurrence

Yes

No
Table 1 Characteristics of 87 patients with locoregional recurrent esophageal cancer (Continued)

\begin{tabular}{lll}
\hline Variables & Number & Percent \\
\hline Treatment moditily after recurrence & 20 & \\
Re-RT only & 7 & 23.0 \\
CRT only & 19 & 21.0 \\
Re-RT concomitant chemotherapy & 39 & 43.7 \\
Best supportive care & 3 & 3.4 \\
Salvage total esophagectomy &
\end{tabular}

\section{Results}

Patient characteristics

The patient characteristics are summarized in Table 1 . The median age was 62 years (range 39-86 years), and the study population included $65(74.7 \%)$ males and 22 (25.3\%) females. Considering KPS at recurrence diagnosis, 32 (36.8\%) of patients were $70-80$, while 55 (63.2\%) were $\geq 80$. Eight (9.2\%) patients had stage I disease, 18 (20.7\%) had stage II, and $61(70.1 \%)$ had stage III at the initial diagnosis. The primary tumor location was the upper thoracic esophagus in $34(39.1 \%)$ patients and the middle and lower thoracic esophagus in $53(60.9 \%)$ patients. The median RFI was 16 months (range 3-168 months), and the RFI was $\leq 12$ months in $38(43.7 \%)$ patients and $>12$ months in 49 (56.3\%) patients. The failure pattern of $62(71.3 \%)$ patients was primary recurrence, $14(16.1 \%)$ cases of regional LN recurrence alone and $11(12.6 \%)$ cases of combined sites. All patients were divided into two groups, 14 patients with regional $L N$ recurrence and the remaining 73 patients with PF. For re-RT patients, 36 of 39 cases received in-field re-irradiation, while the other three cases experienced out-field locoregional failure.

For re-RT patients, the median $\mathrm{BED}_{10}$ of 74.11 Gy (range 48-86.32 Gy) and 60 Gy (range 25.41-84.87 Gy) were delivered in the initial radiation and re-RT,

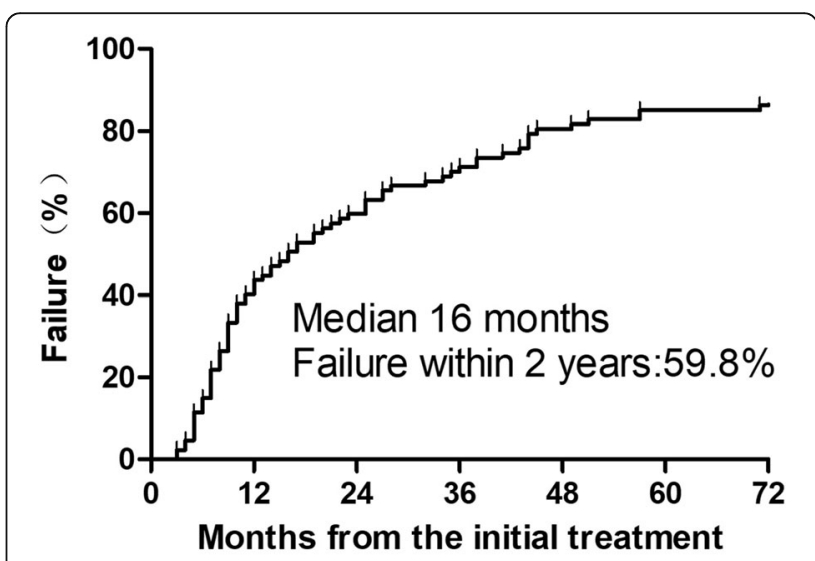

Fig. 1 Time to locoregional recurrence after initial treatment for 87 ESCC patients 
respectively. The median cumulative $\mathrm{BED}_{10}$ was 135.53 Gy (range 96-168 Gy). The median Dmax of spinal cord was 25 Gy (range 9-39 Gy), the median V20 of the total lung and V30 of the heart was 10\% (range $0-24 \%$ ) and 9\% (range 0-25\%), respectively.

\section{OS for the total study population}

The median follow-up was 87 months (range 2206 months). The follow-up rate was $96.6 \%$ (84/87). One patient without re-RT and 7 patients with re-RT remained alive at the last follow-up. For re-RT, 82.1\% (32/39) patients were dead at last follow up, among which, $84.4 \%$ (27/32) patients were cancer-related death. After re-RT, 89.7\% (35/39) suffered from failure, with 24 (68.6\%) cases of distant metastasis alone, 5 (14.3\%) cases of local failure alone and 6 (17.1\%) cases of both. The median survival time (MST) was 10 months (range 1-85 months). The median RFI was 16 months (range 3-168 months). Fifty-two (59.8\%) patients were diagnosed with recurrence within 2 years after initial treatment (Fig. 1).

\section{Propensity score matching and $x^{2}$ tests}

Significant differences in the clinical stage of initial cancer were observed for patients with re-RT $(n=39)$ and without re-RT $(n=48)$ before matching $(P=0.003)$ (Table 2). A nearest neighbor and 1:1 matching algorithm was applied within a default caliper (0.2) [14]. After matching, baseline covariates of the clinicopathological characteristics were corrected, with characteristics being evenly distributed between the re-RT group $(n=33)$ and the non-re-RT group $(n=33$, all $P>0.1)$.

\section{Cox regression analysis for overall sample}

The results of univariate and multivariate analyses for OS are summarized in Table 3. LN recurrence alone and re-RT were associated with better $\mathrm{OS}(P=0.006$ and $P<0.001)$ by Cox univariate analysis. The 1-, 3-, and 5-year OS rates in the $\mathrm{LN}$ group were $84.62 \%, 30.77 \%$, and $23.01 \%$, respectively, and the 1-, 3-, and 5-year OS rates in the PF group were $37.86 \%, 10.29 \%$, and $2.57 \%$, respectively. The MST in the LN group was 23 months, whereas the MST in the PF group was 9 months $(P=0.004$, Fig. 2 a). The 1 -, 3 -, and 5 -year OS rates in the re-RT group were $67.94 \%, 22.89 \%$, and $13.08 \%$, respectively, and the 1-, 3-, and 5-year OS rates for patients without re-RT were $28.52 \%, 6.58 \%$, and $2.19 \%$, respectively. Their MSTs were 21 months and 8 months, respectively $(P<0.001$, Fig. $2 b)$.

Initial clinical stage (I + II vs. III), failure pattern (LN vs. $\mathrm{PF}$ ), re-RT (with vs. without), and chemotherapy for both courses of treatment (with vs. without) were possible prognostic factors in the Cox multivariate model. The failure pattern and re-RT were independent prognostic factors for OS ( $P=0.040$ and $P=0.015$, respectively). However, no statistical difference in OS was observed between the re-RT alone and re-RT with concomitant chemotherapy groups (18 vs. $19, P=0.70$, Fig. 3 ) in the subgroup analysis.
A

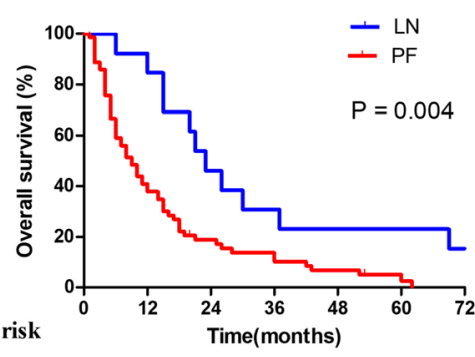

LN

C

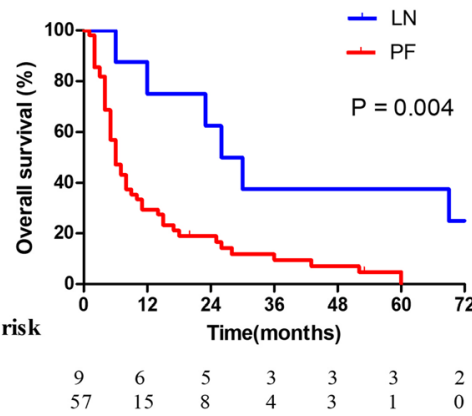

B

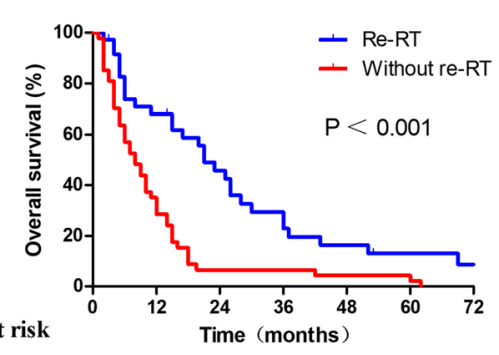

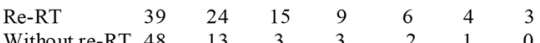

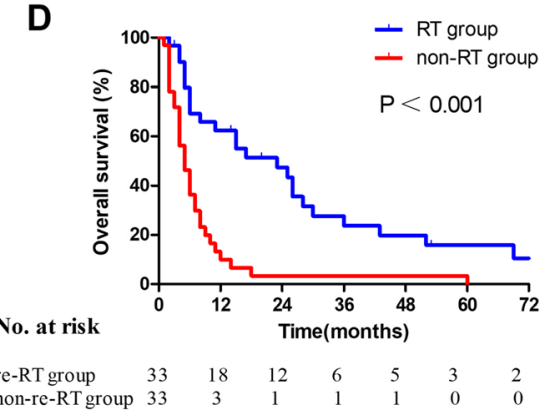

Fig. 2 Kaplan-Meier analysis of OS according to (a) failure pattern (LN vs. PF, $P=0.004$ ) before matching; (b) re-irradiation (re-RT vs. without re-RT, $P<0.001$ ) before matching; (c) failure pattern ( $L N$ vs. $P F, P=0.004$ ) after matching; and (d) re-irradiation (re-RT group vs. non-re-RT group, $P<0.001)$ after matching 
Table 2 Chi-square test of re-RT and without re-RT for locoregional recurrent ESCC before and after matching

\begin{tabular}{|c|c|c|c|c|c|c|}
\hline \multirow[t]{2}{*}{ Variables } & \multicolumn{3}{|c|}{ Before matching } & \multicolumn{3}{|l|}{ After matching } \\
\hline & With re-RT (n) & Without re-RT (n) & P & With re-RT (n) & Without re-RT (n) & $P$ \\
\hline Age (years) & & & 1.000 & & & 1.000 \\
\hline$<65 / \geq 65$ & $26 / 13$ & $32 / 16$ & & $21 / 12$ & $21 / 12$ & \\
\hline KPS & & & 0.770 & & & 0.802 \\
\hline $70-80 />80$ & $15 / 24$ & $17 / 31$ & & $13 / 20$ & $14 / 19$ & \\
\hline Gender & & & 0.572 & & & 0.580 \\
\hline Male/Female & 28/11 & $37 / 11$ & & $23 / 10$ & $25 / 8$ & \\
\hline Smoking & & & 0.619 & & & 0.796 \\
\hline Yes/No & $15 / 24$ & $16 / 32$ & & $12 / 21$ & $11 / 22$ & \\
\hline Alcohol consumption & & & 0.679 & & & 1.000 \\
\hline Yes/No & $6 / 33$ & $9 / 39$ & & $5 / 28$ & $5 / 28$ & \\
\hline Primary tumor location & & & 0.583 & & & 0.802 \\
\hline Upper/Middle and lower thoracic & $14 / 25$ & $20 / 28$ & & $14 / 19$ & $13 / 20$ & \\
\hline Initial clinical stage & & & 0.003 & & & 0.284 \\
\hline$|+\|/\|| \mid$ & $18 / 21$ & $8 / 40$ & & $12 / 21$ & $8 / 25$ & \\
\hline Surgery in the initial treatment & & & 0.488 & & & 0.392 \\
\hline Yes/No & $6 / 33$ & $5 / 43$ & & $2 / 31$ & $4 / 29$ & \\
\hline Chemotherapy in the initial treatment & & & 0.582 & & & 0.806 \\
\hline Yes/No & $18 / 21$ & $25 / 23$ & & $16 / 17$ & $17 / 16$ & \\
\hline Radiation dose in the initial treatment (Gy) & & & 0.101 & & & $0.213^{*}$ \\
\hline$\leq 50 />50$ & $8 / 31$ & $4 / 44$ & & $5 / 28$ & $2 / 31$ & \\
\hline Recurrence-free interval (months) & & & 0.187 & & & 0.215 \\
\hline Median (range) & $27(4163)$ & $12.5(3168)$ & & $27(5163)$ & 12(3144) & \\
\hline$\leq 12 />12$ & $14 / 25$ & $24 / 24$ & & $12 / 21$ & $17 / 16$ & \\
\hline
\end{tabular}

Abbreviation: Re-RT Re-irradiation, KPS Karnofsky performance status. *:Fisher's exact tests

\section{Cox regression analysis for matched cohort}

In the matched cohort, failure pattern and re-RT were independently associated with OS for recurrent ESCC $(P=0.025$ and $P=0.002$, respectively; Table 4$)$. For the two failure patterns (LN vs. PF), the comparative 1-, 3-, and 5 -year OS rates were $75.00 \%$ vs. $29.49 \%, 37.50 \%$ vs. $9.53 \%$, and $37.50 \%$ vs. $0 \%$, respectively. The MSTs in the LN and PF groups were 28 months and 6 months, respectively $(P=0.004$, Fig. $2 \mathrm{c})$. For treatment with re-RT or no re-RT (with or without), the comparative 1-, 3-, and 5 -year OS rates were $62.38 \%$ vs. $9.93 \%, 23.71 \%$ vs. $3.31 \%$, and $15.81 \%$ vs. $0 \%$, respectively. The MSTs in the re-RT and without re-RT groups were 23 months and 5 months, respectively $(P<0.001$, Fig. 2 d).

\section{Toxicity}

In the re-RT and non-re-RT groups of the matched cohort, $9.09 \%(3 / 33)$ and $3.03 \%(1 / 33)$ of cases experienced TEF, $15.15 \%(5 / 33)$ and $3.03 \%(1 / 33)$ of cases experienced pericardial/pleural effusion $(P=0.613$ and $P=0.197$, respectively). The rates of grade $3 \mathrm{RP}$ were $24.24 \%(8 / 33)$ and $6.06 \%(2 / 33)$ in the re-RT and
non-re-RT groups, respectively $(P=0.039)$. No case of grade $5 \mathrm{RP}$ was observed. The median age of the $10 \mathrm{pa}-$ tients who developed RP was 61 years (range $43-83$ years). The radiation doses for primary RT in 2 patients not treated with re-RT were 63 Gy and 70 Gy. The median doses for primary RT and re-RT in the other eight patients were 62.2 Gy (range 41-64 Gy) and 50.3 Gy (range 36-60 Gy), respectively. No significant correlation was found between RP and the V20 of the total lungs in re-RT $(P=0.25)$. No treatment-related deaths were recorded.

\section{Discussion}

Locoregional recurrence occurs frequently after primary definitive RT or multimodal therapy for ESCC. Yet, therapeutic options remain limited, and no consensus regarding the optimal treatment has been reached. Re-RT for the management of recurrent ESCC is one of the options, and in the present study, the effectiveness and toxicity of re-RT were retrospectively analyzed via PSM analysis. In the whole cohort, the failure pattern and re-RT were found to be independent prognostic 
Table 3 Cox model analysis for 87 ESCC patients with locoregional recurrence before matching

\begin{tabular}{|c|c|c|c|c|c|c|c|}
\hline \multirow[t]{2}{*}{ Variable } & \multirow[t]{2}{*}{$\mathrm{n}$} & \multicolumn{3}{|c|}{ Univariate } & \multicolumn{3}{|c|}{ Multivariate } \\
\hline & & $\mathrm{HR}$ & $95 \% \mathrm{Cl}$ & $P$ & $\mathrm{HR}$ & $95 \% \mathrm{Cl}$ & $P$ \\
\hline \multicolumn{8}{|l|}{ Age (years) } \\
\hline$<65 / \geq 65$ & $58 / 29$ & 0.786 & $0.467-1.322$ & 0.364 & & & \\
\hline \multicolumn{8}{|l|}{ KPS } \\
\hline $70-80 />80$ & $32 / 55$ & 0.752 & $0.467-1.212$ & 0.243 & & & \\
\hline \multicolumn{8}{|l|}{ Gender } \\
\hline Male/Female & $65 / 22$ & 1.008 & $0.607-1.673$ & 0.976 & & & \\
\hline \multicolumn{8}{|l|}{ Smoking } \\
\hline Yes/No & $31 / 56$ & 1.438 & $0.893-2.317$ & 0.135 & & & \\
\hline \multicolumn{8}{|l|}{ Alcohol consumption } \\
\hline Yes/No & $15 / 72$ & 1.229 & $0.668-2.261$ & 0.507 & & & \\
\hline \multicolumn{8}{|l|}{ Primary tumor location } \\
\hline Upper/Middle and lower thoracic & $34 / 53$ & 1.241 & $0.779-1.977$ & 0.364 & & & \\
\hline \multicolumn{8}{|l|}{ Clinical stage } \\
\hline$|+\|/\|| \mid$ & $26 / 61$ & 1.083 & $0.645-1.820$ & 0.763 & 1.027 & $0.599-1.761$ & 0.923 \\
\hline \multicolumn{8}{|l|}{ Surgery in the initial treatment } \\
\hline Yes/No & $11 / 76$ & 1.161 & $0.608-2.216$ & 0.652 & & & \\
\hline \multicolumn{8}{|l|}{ Chemotherapy in the initial treatment } \\
\hline Yes/No & $43 / 44$ & 0.954 & $0.601-1.514$ & 0.842 & & & \\
\hline \multicolumn{8}{|l|}{ Recurrence-free interval (months) } \\
\hline$\leq 12 />12$ & $38 / 49$ & 0.884 & $0.558-1.402$ & 0.601 & & & \\
\hline \multicolumn{8}{|l|}{ Pattern of recurrence } \\
\hline LN/PF & $14 / 73$ & 0.385 & $0.195-0.762$ & 0.006 & 0.461 & $0.221-0.964$ & 0.040 \\
\hline \multicolumn{8}{|l|}{ Re-RT after recurrence } \\
\hline Yes/No & $39 / 48$ & 0.392 & $0.239-0.642$ & $<0.001$ & 0.513 & $0.299-0.878$ & 0.015 \\
\hline \multicolumn{8}{|l|}{ Chemotherapy after recurrence } \\
\hline Yes/No & $26 / 61$ & 0.799 & $0.478-1.335$ & 0.391 & & & \\
\hline \multicolumn{8}{|c|}{ Chemotherapy for both course treatment } \\
\hline Yes/No & $13 / 74$ & 0.540 & $0.274-1.063$ & 0.074 & 0.520 & $0.257-1.051$ & 0.069 \\
\hline
\end{tabular}

Abbreviations: HR Hazard ratio, 95\%Cl 95\% confidence interval, Re-RT Re-irradiation, LN Regional lymph node recurrence only, PF Primary failure with/without regional lymph node recurrence, KPS Karnofsky performance status

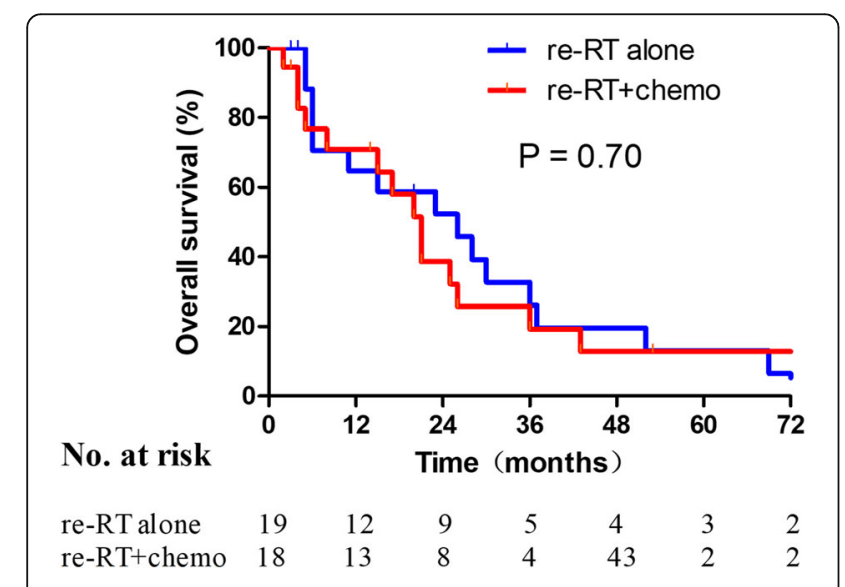

Fig. 3 Patient survival after re-RT with or without chemotherapy factors for OS $(P=0.040$ and $P=0.015$, respectively), and these results were also verified in the two well-balanced groups after propensity score matching. Furthermore, significant differences in OS and MST were observed for different failure patterns (LN vs. PF, MST 28 months vs. 6 months, $P=0.004$ ) as well as for re-RT (re-RT vs. non-re-RT, MST 23 months vs. 5 months, $P<0.001$ ).

The current study showed that in the majority of cases (59.8\%), locoregional recurrence occurred within 2 years after initial treatment. The median RFI was 16 months (range 3-168 months), which was similar to the results of Chen et al. [15]. PF was the most common (71.3\%) failure pattern, followed by regional LN alone (16.1\%) 
Table 4 Cox model analysis for 66 ESCC patients with locoregional recurrence after matching

\begin{tabular}{|c|c|c|c|c|c|c|c|}
\hline \multirow[t]{2}{*}{ Variable } & \multirow[t]{2}{*}{$\mathrm{n}$} & \multicolumn{3}{|c|}{ Univariate } & \multicolumn{3}{|c|}{ Multivariate } \\
\hline & & $\overline{H R}$ & $95 \% \mathrm{Cl}$ & $P$ & $\overline{H R}$ & $95 \% \mathrm{Cl}$ & $P$ \\
\hline \multicolumn{8}{|l|}{ Age (years) } \\
\hline$<65 / \geq 65$ & $42 / 24$ & 0.736 & $0.407-1.330$ & 0.310 & & & \\
\hline \multicolumn{8}{|l|}{ KPS } \\
\hline $70-80 />80$ & $27 / 39$ & 0.843 & $0.491-1.447$ & 0.535 & & & \\
\hline \multicolumn{8}{|l|}{ Gender } \\
\hline Male/Female & $48 / 18$ & 0.942 & $0.530-1.674$ & 0.838 & & & \\
\hline \multicolumn{8}{|l|}{ Smoking } \\
\hline Yes/No & $23 / 43$ & 1.486 & $0.857-2.577$ & 0.159 & & & \\
\hline \multicolumn{8}{|l|}{ Alcohol consumption } \\
\hline Yes/No & $10 / 56$ & 1.083 & $0.526-2.233$ & 0.828 & & & \\
\hline \multicolumn{8}{|l|}{ Primary tumor location } \\
\hline Upper/Middle and lower thoracic & $27 / 39$ & 1.265 & $0.733-2.183$ & 0.398 & & & \\
\hline \multicolumn{8}{|l|}{ Clinical stage } \\
\hline$|+||/|||$ & $20 / 46$ & 1.021 & $0.561-1.858$ & 0.946 & 1.541 & $0.814-2.916$ & 0.184 \\
\hline \multicolumn{8}{|l|}{ Surgery in the initial treatment } \\
\hline Yes/No & $6 / 60$ & 2.258 & $0.942-5.414$ & 0.068 & & & \\
\hline \multicolumn{8}{|l|}{ Chemotherapy in the initial treatment } \\
\hline Yes/No & $33 / 33$ & 1.005 & $0.585-1.729$ & 0.984 & & & \\
\hline \multicolumn{8}{|l|}{ Recurrence-free interval (months) } \\
\hline$\leq 12 />12$ & $29 / 37$ & 0.739 & $0.433-1.262$ & 0.268 & & & \\
\hline \multicolumn{8}{|l|}{ Pattern of recurrence } \\
\hline LN/PF & $9 / 57$ & 0.277 & $0.108-0.714$ & 0.008 & 0.319 & $0.117-0.869$ & 0.025 \\
\hline \multicolumn{8}{|l|}{ Re-RT after recurrence } \\
\hline Yes/No & $33 / 33$ & 0.299 & $0.167-0.535$ & $<0.001$ & 0.375 & $0.201-0.701$ & 0.002 \\
\hline \multicolumn{8}{|l|}{ Chemotherapy after recurrence } \\
\hline Yes/No & $20 / 46$ & 0.817 & $0.456-1.463$ & 0.497 & & & \\
\hline \multicolumn{8}{|c|}{ Chemotherapy for both course treatment } \\
\hline Yes/No & $10 / 56$ & 0.621 & $0.291-1.323$ & 0.217 & 0.710 & $0.323-1.562$ & 0.395 \\
\hline
\end{tabular}

Abbreviations: HR Hazard ratio, 95\%Cl 95\% confidence interval, Re-RT Re-irradiation, LN Regional lymph node recurrence alone, $P F$ Primary failure with/without regional lymph node recurrence, KPS Karnofsky performance status

and both (12.6\%). This distribution deviated slightly from that in a previous study by Versteijne et al., which was $57 \%, 14 \%$ and $29 \%$ respectively [16]. This might be attributed to differences in the pathological composition of the tumors or radiation doses given for initial treatment. Also, in the current study, failure pattern (LN vs. $\mathrm{PF}$ ) was an independent prognostic factor for OS. PF indicated a worse OS compared to $\mathrm{LN}(P=0.004, \mathrm{HR}=$ 0.3754, 95\% confidence interval [CI] 0.1939-0.7266), which emphasized that good control of the primary tumor plays a vital role in ESCC management.

Patients with recurrent ESCC previously treated with RT who are in good clinical condition could be selected for potentially curative treatment. Previous study had reported encouraging outcomes of re-RT for symptoms relief [17], in which 4 had complete resolution and 4 had diminished or stable symptoms among the 10 patients who presented with symptomatic disease. Moreover, Zhou et al. [18] reported that the 3-years OS for primary tumor recurrent ESCC was $21.8 \%$ with a MST of 20 months upon salvage RT group. Similarly, the 3-years OS was $22.89 \%$ among our re-RT patients with a MST of 21 months. The re-RT group had a significantly higher OS compared to the non-re-RT group in the current matched cohort $(P<0.001, \mathrm{HR}=0.2426,95 \% \mathrm{CI}$ 0.1294-0.4547). Yamashita et al. [19] reported a MST of 13.8 months for locoregional recurrent ESCC patients with re-RT. This inferior MST might be related to 
differences in the recurrent tumor location and initial treatment baseline characteristics. Salvage doses of re-irradiation should be delivered to patients with localized disease to improve local control and OS.

Concurrent CRT is the standard treatment for ESCC patients who decline or cannot tolerate surgery. However, no evidence of survival benefits from concurrent CRT was found. Concurrent CRT was shown to cause severe acute esophagitis in $15-25 \%$ of thoracic radiotherapy cases [20]. In addition, most cases of recurrent ESCC occurred in older patients for whom concurrent CRT might be sub-optimal. In the current subgroup analysis, no statistical difference in OS was found between the groups treated with re-RT alone and re-RT combined with chemotherapy $(P=0.70)$. Also, two of three cases suffered from TEF upon concurrent CRT. Thus, concurrent CRT might increase toxicity without a survival benefit.

Concerning the potentially serious complications, re-RT was performed in a small and highly selected group of patients in clinical practice. In a prospective and randomized study, which included 34 patients who received re-RT and 35 patients who received dilatation alone, 6 cases of TEF were observed in the non-re-RT group, while no case of TEF was found in the re-RT group [21]. In the current study, no statistical differences were found in the incidence of TEF $(P=0.613)$ and pericardial/pleural effusion $(P=0.197)$ between re-RT and non-re-RT groups. As reported by Yamaguchi et al. [19], advanced T stage (T3 or T4) at the recurrence diagnosis was significantly associated with grade 3 or above toxicities. This might imply that TEF might associated with tumor progression. However, the impact of repair disability for re-irradiated tissues should also be considered.

$\mathrm{RP}$ is another concern in thoracic re-RT. Sumita et al. [22] had retrospectively analyzed 21 lung cancer patients who underwent X-ray beam re-RT and only one grade 3 $\mathrm{RP}$ was observed. The incidence of grade $3 \mathrm{RP}$ was $24.24 \%$ for re-RT group in our study, but even with this high incidence of RP, no pneumonia-related deaths occurred. There was no correlation between RP and the V20 of the total lungs in the present study, which might relate to the limited sample, the different initial radiation schedules and interval. In addition, Ren et al. [23] showed that both re-RT and initial-RT influenced the incidence of grade 3 or above RP. However, further studies concerning the toxicities of the OARs are required.

As a retrospective study, records for symptoms such as dysphagia, weight loss, hoarseness, and cough were not available, and thus, symptom control was not evaluated in the present study. Moreover, because this was a single-center study, the number of cases was limited due to the rarity of re-RT treatment. Therefore, the implications of the findings could be limited.

\section{Conclusions}

Re-RT was feasible and beneficial for locoregional recurrent ESCC patients after primary RT. Compared to CRT, re-RT alone is more appropriate. Long-term survival was improved with re-RT. Despite a high incidence of RP, toxicities were tolerable.

\begin{abstract}
Abbreviations
5-FU: 5-flurouracil; AJCC: American Joint Committee on Cancer: CRT: Chemoradiotherapy; CT: Computed tomography; CTCAE: National Cancer Institute Common Terminology Criteria for Adverse Events; ESCC: Esophageal squamous cell carcinoma; EUS: Endoscopic ultrasound; HR: Hazard ratios; KPS: Karnofsky performance status; LN: Regional lymph node recurrence only; MST: Median survival time; OS: Overall survival; PF: Primary failure with/without regional lymph node recurrence; PSM: Propensity score-matched; Re-RT: Re-irradiation; RFI: Recurrence-free interval; RP: Radiation pneumonitis; TEF: Tracheoesophageal fistula
\end{abstract}

\section{Funding}

This study was supported by The Fujian Province Natural Science Foundation (2016 J01437 and 2017 J01260), The Fujian Medical Innovation Project (2015-CX-8), Joint Funds for the Innovation of Science and Technology, Fujian province (2017Y9074) and Peking University Cancer Hospital \& Institute, Key Laboratory of Carcinogenesis and Translational Research, Ministry of Education/Beijing (2017 Open Project-9).

\section{Availability of data and materials}

All data generated or analyzed during this study are included in this published article.

\section{Authors' contributions}

All authors helped to perform the research; LH manuscript writing and performing procedures; YXH manuscript writing and data analysis; QYZ, XQZ and LRT contribution to drafting conception and design; KXD, XYL and BHZ contribution to writing the manuscript, JXW and SLC participated in data analysis; JLL contribution to writing the manuscript, drafting conception and design. All authors approved the final manuscript.

Ethics approval and consent to participate

This study was approved by the Ethics Committee of Fujian Medical University Cancer Hospital, Fuzhou, China (KT2018-006-01).

Consent for publication

Not applicable.

Competing interests

The authors declare that they have no competing interests.

\section{Publisher's Note}

Springer Nature remains neutral with regard to jurisdictional claims in published maps and institutional affiliations.

\section{Author details}

${ }^{1}$ Department of Radiation Oncology, Fujian Medical University Cancer Hospital, Fujian Cancer Hospital, Fuzhou 350014, China. ${ }^{2}$ Department of Radiation Oncology, Xiamen Humanity Hospital, Xiamen, China. ${ }^{3}$ Biomedical Research Center of South China, Fujian Normal University, Fuzhou, China.

Received: 10 July 2018 Accepted: 30 August 2018

Published online: 10 September 2018

\section{References}

1. Welsh J, Settle SH, Amini A, Xiao L, Suzuki A, Hayashi Y, et al. Failure patterns in patients with esophageal cancer treated with definitive chemoradiation. Cancer. 2012;118(10):2632-40. [PMID:PMC3747650 10.1002/ cncr.26586]

2. Pennathur A, Gibson MK, Jobe BA, Luketich JD. Oesophageal carcinoma. Lancet. 2013;381(9864):400-12. 10.1016/S0140-6736(12)60643-6] 
3. Shioyama Y, Nakamura K, Ohga S, Nomoto S, Sasaki T, Yamaguchi T, et al. Radiation therapy for recurrent esophageal cancer after surgery: clinical results and prognostic factors. Jpn J Clin Oncol. 2007;37(12):918-23. 10. 1093/jjco/hym138]

4. Markar SR, Karthikesalingam A, Penna M, Low DE. Assessment of short-term clinical outcomes following salvage esophagectomy for the treatment of esophageal malignancy: systematic review and pooled analysis. Ann Surg Oncol. 2014;21(3):922-31. 10.1245/s10434-013-3364-0]

5. Swisher SG, Wynn P, Putnam JB, Mosheim MB, Correa AM, Komaki RR, et al. Salvage esophagectomy for recurrent tumors after definitive chemotherapy and radiotherapy. J Thorac Cardiovasc Surg. 2002;123(1):175-83.

6. Bots WTC, van den Bosch S, Zwijnenburg EM, Dijkema T, van den Broek GB, Weijs WLJ, et al. Reirradiation of head and neck cancer: Long-term disease control and toxicity. Head Neck. 2017;39(6):1122-30. [PMID:PMC5485062 10. 1002/hed.24733]

7. Chao HH, Berman AT, Simone CB 2nd, Ciunci C, Gabriel P, Lin H, et al. Multiinstitutional prospective study of Reirradiation with proton beam radiotherapy for Locoregionally recurrent non-small cell lung Cancer. J Thorac Oncol. 2017;12(2):281-92. 10.1016/j.jtho.2016.10.018]

8. Zemlin A, Martens B, Wiese B, Merten R, Steinmann D. Timing of reirradiation in recurrent high-grade gliomas: a single institution study. J Neurooncol. 2018;138(3):571-9. https://doi.org/10.1007/s11060-018-2824-6.

9. Kim YS. Reirradiation of head and neck cancer in the era of intensitymodulated radiotherapy: patient selection, practical aspects, and current evidence. Radiat Oncol J. 2017;35(1):1-15. [PMID:PMC5398346 10.3857/roj. 2017.00122]

10. Nieder C, De Ruysscher D, Gaspar LE, Guckenberger M, Mehta MP, Cheung $P$, et al. Reirradiation of recurrent node-positive non-small cell lung cancer after previous stereotactic radiotherapy for stage I disease : a multiinstitutional treatment recommendation. Strahlenther Onkol. 2017;193(7): 515-24. 10.1007/s00066-017-1130-0]

11. Tao R, Tsai CJ, Jensen G, Eng C, Kopetz S, Overman MJ, et al. Hyperfractionated accelerated reirradiation for rectal cancer: an analysis of outcomes and toxicity. Radiother Oncol. 2017;122(1):146-51. 10.1016/j. radonc.2016.12.015]

12. Chen HY, Ma XM, Ye M, Hou YL, Xie HY, Bai YR. Esophageal perforation during or after conformal radiotherapy for esophageal carcinoma. J Radiat Res. 2014;55(5):940-7. [PMID:PMC4202289 10.1093/jrr/rru031]

13. Graves PR, Siddiqui F, Anscher MS, Movsas B. Radiation pulmonary toxicity: from mechanisms to management. Semin Radiat Oncol. 2010;20(3):201-7. 10.1016/j.semradonc.2010.01.010]

14. Huang F, Du C, Sun M, Ning B, Luo Y, An S. Propensity score matching in SPSS. Nan Fang Yi Ke Da Xue Xue Bao. 2015;35(11):1597-601.

15. Chen $Y, L$ Lu $Y$, Wang $Y$, Yang $H, X i a ~ Y, C h e n ~ M$, et al. Comparison of salvage chemoradiation versus salvage surgery for recurrent esophageal squamous cell carcinoma after definitive radiochemotherapy or radiotherapy alone. Dis Esophagus : official journal of the International Society for Diseases of the Esophagus. 2014;27(2):134-40. 10.1111/j.1442-2050.2012.01440.x]

16. Versteijne $\mathrm{E}$, van Laarhoven HW, van Hooft JE, van Os RM, Geijsen ED, van Berge Henegouwen Ml, et al. Definitive chemoradiation for patients with inoperable and/or unresectable esophageal cancer: locoregional recurrence pattern. Dis Esophagus : official journal of the International Society for Diseases of the Esophagus. 2015;28(5):453-9. 10.1111/dote.12215]10.1111/ dote.12215]

17. Fernandes A, Berman AT, Mick R, Both S, Lelionis K, Lukens JN, et al. A prospective study of proton beam Reirradiation for esophageal Cancer. Int J Radiat Oncol Biol Phys. 2016;95(1):483-7. 10.1016/j.ijrobp.2015.12.005]

18. Zhou ZG, Zhen CJ, Bai WW, Zhang P, Qiao XY, Liang JL, et al. Salvage radiotherapy in patients with local recurrent esophageal cancer after radical radiochemotherapy. Radiat Oncol. 2015;10:54. [PMID:PMC4351944 10.1186/ s13014-015-0358-z]

19. Yamaguchi S, Ohguri T, Imada H, Yahara K, Moon SD, Higure A, et al. Multimodal approaches including three-dimensional conformal reirradiation for recurrent or persistent esophageal cancer: preliminary results. J Radiat Res. 2011;52(6):812-20.

20. Werner-Wasik M, Pequignot E, Leeper D, Hauck W, Curran W. Predictors of severe esophagitis include use of concurrent chemotherapy, but not the length of irradiated esophagus: a multivariate analysis of patients with lung cancer treated with nonoperative therapy. Int J Radiat Oncol Biol Phys. 2000;48(3):689-96
21. Teli MA, Mushood GN, Zargar SA, Andrabi WH. Comparative evaluation between re-irradiation and demand endoscopic dilatation vs endoscopic dilatation alone in patients with recurrent/reactivated residual in-field esophageal malignancies. J Cancer Res Ther. 2008;4(3):121-5.

22. Sumita K, Harada H, Asakura H, Ogawa H, Onoe T, Murayama S, et al. Reirradiation for locoregionally recurrent tumors of the thorax: a singleinstitution, retrospective study. Radiat Oncol. 2016;11:104. [PMID: PMC4971641 10.1186/s13014-016-0673-z]

23. Ren C, Ji T, Liu T, Dang J, Li G. The risk and predictors for severe radiation pneumonitis in lung cancer patients treated with thoracic reirradiation. Radiat Oncol. 2018;13(1):69. [PMID:PMC5902864 10.1186/s13014-018-1016-z]

\section{Ready to submit your research? Choose BMC and benefit from:}

- fast, convenient online submission

- thorough peer review by experienced researchers in your field

- rapid publication on acceptance

- support for research data, including large and complex data types

- gold Open Access which fosters wider collaboration and increased citations

- maximum visibility for your research: over $100 \mathrm{M}$ website views per year

At BMC, research is always in progress.

Learn more biomedcentral.com/submissions 